DESY 94-235

December 1994

\title{
Gluon Radiation Off Scalar Stop Particles
}

\author{
W. Beenakker, R. Höpker and P. M. Zerwas \\ Deutsches Elektronen-Synchrotron DESY, D-22603 Hamburg, FRG
}

\begin{abstract}
We present the distributions for gluon radiation off stop-antistop particles produced in $e^{+} e^{-}$ annihilation: $e^{+} e^{-} \rightarrow \tilde{t} \tilde{\tilde{t}} g$. For high energies the splitting functions of the fragmentation processes $\tilde{t} \rightarrow \tilde{t} g$ and $g \rightarrow \tilde{t} \overline{\tilde{t}}$ are derived; they are universal and apply also to high-energy stop particles produced at hadron colliders.
\end{abstract}


Introduction. Stop particles are exceptional among the supersymmetric partners of the standard-model fermions. Since the top quarks are heavy, the masses of the two stop particles $\tilde{t}_{1}$ and $\tilde{t}_{2}$, mixtures of the left $(\mathrm{L})$ and right $(\mathrm{R})$ squarks, may split into two levels separated by a large gap [1]-[阿. The mass of the lightest eigenstate $\tilde{t}_{1}$ could be so low that the particle may eventually be accessible at the existing $p \bar{p}$ and even $e^{+} e^{-}$storage rings. So far the result of search experiments at $e^{+} e^{-}$colliders [4, 5] has been negative and a lower limit of $45.1 \mathrm{GeV}$ has been set at LEP [5] for the L/R mixing angle outside the band of $\cos ^{2} \theta_{t}$ between 0.17 and 0.44 and for a mass difference between the $\tilde{t}_{1}$ and the lightest neutralino $\tilde{\chi}_{1}^{0}$ of more than $5 \mathrm{GeV}$. The higher energy at LEP2 and dedicated efforts at the Tevatron will open the mass range beyond the current limits soon.

To begin, we briefly summarize the well-known theoretical predictions for the cross section of the production process [Fig. [1(a)]

$$
e^{+} e^{-} \rightarrow \tilde{t}_{1} \overline{\tilde{t}}_{1}
$$

For a given value $\theta_{t}$ of the $\mathrm{L} / \mathrm{R}$ mixing angle, the vertices of the $\tilde{t}_{1}$ pair with the photon and the $Z$ boson may be written as $i e_{0} \tilde{Q}\left[p_{\tilde{t}_{1}}-p_{\tilde{t}_{1}}\right]_{\mu}$, where $p_{\tilde{t}_{1}}$ and $p_{\tilde{t}_{1}}$ are the 4-momenta of the stop and antistop squarks, and the charges read

$$
\begin{aligned}
\tilde{Q}_{\gamma} & =-e_{t} \\
\tilde{Q}_{Z} & =\left(\cos ^{2} \theta_{t}-2 e_{t} \sin ^{2} \theta_{W}\right) / \sin 2 \theta_{W}
\end{aligned}
$$

respectively. $\theta_{W}$ is the standard electroweak mixing angle and $e_{0}=\sqrt{4 \pi \alpha}$ is the electromagnetic coupling to be evaluated with $\alpha^{-1}\left(M_{Z}\right)=129.1$ in the improved Born approximation [6]. The $Z$ boson coupling vanishes for the $\mathrm{L} / \mathrm{R}$ mixing angle $\cos ^{2} \theta_{t} \rightarrow 2 e_{t} \sin ^{2} \theta_{W} \approx 0.30$. Defining the $\gamma$ and $Z$ vector/axial-vector charges of the electron, as usual, by $e_{e}=-1$, $v_{e}=-1+4 \sin ^{2} \theta_{W}$ and $a_{e}=-1$, the cross section can be expressed in the compact form [2]

$$
\begin{aligned}
\sigma_{B}\left[e^{+} e^{-} \rightarrow \tilde{t}_{1} \overline{\tilde{t}}_{1}\right]=\frac{\pi \alpha^{2}}{s}\left[\tilde{Q}_{\gamma}^{2}\right. & +\frac{\left(v_{e}^{2}+a_{e}^{2}\right) \tilde{Q}_{Z}^{2}}{4 \sin ^{2} 2 \theta_{W}} \frac{s^{2}}{\left(s-M_{Z}^{2}\right)^{2}+M_{Z}^{2} \Gamma_{Z}^{2}} \\
& \left.+\frac{v_{e} \tilde{Q}_{\gamma} \tilde{Q}_{Z}}{\sin 2 \theta_{W}} \frac{s\left(s-M_{Z}^{2}\right)}{\left(s-M_{Z}^{2}\right)^{2}+M_{Z}^{2} \Gamma_{Z}^{2}}\right] \beta^{3}
\end{aligned}
$$

where $\sqrt{s}$ is the center of mass energy and $M_{Z}, \Gamma_{Z}$ are the mass and the total width of the $Z$ boson, respectively. The $P$-wave excitation near the threshold gives rise to the familiar $\beta^{3}$ suppression, where $\beta=\left(1-4 m_{\tilde{t}_{1}}^{2} / s\right)^{1 / 2}$ is the velocity of the stop particles. Angular momentum conservation enforces the $\sin ^{2} \theta$ law, $\sigma_{B}^{-1} d \sigma_{B} / d \cos \theta=\frac{3}{4} \sin ^{2} \theta$, for the angular distribution of the stop particles with respect to the beam axis.

QCD corrections. Gluonic corrections modify the cross section [7, 8] The virtual corrections, Fig.](b), can be expressed by the form factor

\footnotetext{
${ }^{1}$ Since we focus on QCD gluon effects for light stop particles in the LEP range, we do not take into account quark-gluino loop effects, assuming the gluino to be heavy; these loop effects have been discussed for squark production at the Tevatron in Ref. 9] and at $e^{+} e^{-}$colliders in Ref.[10].
} 


$$
\begin{aligned}
F(s)=\frac{4}{3} \frac{\alpha_{s}}{\pi}\left\{\frac { s - 2 m _ { \tilde { t } _ { 1 } } ^ { 2 } } { s \beta } \left[2 \operatorname{Li}_{2}(w)+2 \log (w) \log (1-w)-\frac{1}{2} \log ^{2}(w)+\frac{2}{3} \pi^{2}-2 \log (w)\right.\right. \\
\left.\left.-\log (w) \log \left(\frac{\lambda^{2}}{m_{\tilde{t}_{1}}^{2}}\right)\right]-2-\log \left(\frac{\lambda^{2}}{m_{\tilde{t}_{1}}^{2}}\right)\right\}
\end{aligned}
$$

where $\alpha_{s}$ is the strong coupling constant and the kinematical variable $w$ is defined as $w=$ $(1-\beta) /(1+\beta)$. The form factor is infrared (IR) divergent. We have regularized this divergence by introducing a small parameter $\lambda$ for the gluon mass. The IR singularity is eliminated by adding the contribution of the soft gluon radiation [Fig.11(c)], with the scaled gluon energy integrated up to a cut-off value $\epsilon_{g}=2 E_{g}^{c u t} / \sqrt{s} \ll 1$. The sum of the virtual correction $(V)$ and the soft-gluon radiation $(S)$ depends only on the physical energy cut-off $\epsilon_{g}$

$$
\begin{aligned}
\sigma_{V+S}= & \sigma_{B} \frac{4}{3} \frac{\alpha_{s}}{\pi}\left\{\frac { s - 2 m _ { \tilde { t } _ { 1 } } ^ { 2 } } { s \beta } \left[4 \operatorname{Li}_{2}(w)-2 \log (w) \log (1+w)+4 \log (w) \log (1-w)\right.\right. \\
& \left.\left.+\frac{1}{3} \pi^{2}-2 \log (w) \log \left(\epsilon_{g}\right)\right]+\frac{4 m_{\tilde{t}_{1}}^{2}-3 s}{s \beta} \log (w)+\log \left(\frac{m_{\tilde{t}_{1}}^{2}}{s}\right)-2 \log \left(\epsilon_{g}\right)-2\right\}
\end{aligned}
$$

After including the hard gluon radiation, the dependence on the cut-off $\epsilon_{g}$ disappears from the total cross section. The total QCD corrections can finally be summarized in a universal factor 8

$$
\sigma\left[e^{+} e^{-} \rightarrow \tilde{t}_{1} \overline{\tilde{t}}_{1}(g)\right]=\sigma_{B}\left[1+\frac{4}{3} \frac{\alpha_{s}}{\pi} f(\beta)\right]
$$

with (Fig.2)

$$
\begin{aligned}
f(\beta)= & \frac{1+\beta^{2}}{\beta}\left[4 \operatorname{Li}_{2}(w)+2 \operatorname{Li}_{2}(-w)+2 \log (w) \log (1-w)+\log (w) \log (1+w)\right] \\
& -4 \log (1-w)-2 \log (1+w)+\left[3+\frac{1}{\beta^{3}}\left(2-\frac{5}{4}\left(1+\beta^{2}\right)^{2}\right)\right] \log (w)+\frac{3}{2} \frac{1+\beta^{2}}{\beta^{2}}
\end{aligned}
$$

Very close to the threshold the Coulombic gluon exchange between the slowly moving stop particles generates the universal Sommerfeld rescattering singularity [11] $f \rightarrow \pi^{2} / 2 \beta$, which damps the threshold suppression, yet does not neutralize it entirely. Employing methods based on non-relativistic Green's functions, an adequate description of stop pair production near threshold has been given in Ref. [12], which also takes into account screening effects due to the finite decay width of the stop particles. In the high-energy limit [8] the correction factor in eq.(3) approaches the value $\left(1+4 \alpha_{s} / \pi\right)$.

In this note we present a general analysis of hard gluon radiation. We also include stop fragmentation due to collinear gluon emission in the perturbative regime at high energies and we give an account of non-perturbative fragmentation effects. 
For unpolarized lepton beams the cross section for gluon radiation off $\tilde{t}_{1}$ squarks

$$
e^{+} e^{-} \rightarrow \tilde{t}_{1} \overline{\tilde{t}}_{1} g
$$

depends on four variables: the polar angle $\theta$ between the momentum of the $\tilde{t}_{1}$ squark and the $e^{-}$momentum, the azimuthal angle $\chi$ between the $\tilde{t}_{1} \overline{\tilde{t}}_{1} g$ plane and the plane spanned by the $e^{ \pm}$beam axis with the $\tilde{t}_{1}$ momentum [see Ref.[13]], and two of the scaled energies $x\left(\tilde{t}_{1}\right)$, $\bar{x}\left(\tilde{\tilde{t}}_{1}\right), z(g)$ in units of the beam energy. The energies are related through $x+\bar{x}+z=2$ and vary over the intervals $\mu \leq x, \bar{x} \leq 1$ and $0 \leq z \leq 1-\mu^{2}$, where $\mu=2 m_{\tilde{t}_{1}} / \sqrt{s}$ denotes the squark mass in units of the beam energy. For the angles between the squark and gluon momenta we have

$$
\begin{aligned}
\cos \theta_{\tilde{t}_{1} \tilde{t}_{1}} & =\frac{2-2(x+\bar{x})+x \bar{x}+\mu^{2}}{\sqrt{\left(x^{2}-\mu^{2}\right)\left(\bar{x}^{2}-\mu^{2}\right)}} \\
\cos \theta_{\tilde{t}_{1} g} & =\frac{2-2(x+z)+x z}{z \sqrt{x^{2}-\mu^{2}}}
\end{aligned}
$$

The spin-1 helicity analysis of the cross section results in the following well-known angular decomposition 14

$$
\begin{aligned}
\frac{d \sigma}{d x d \bar{x} d \cos \theta d \chi / 2 \pi}= & \frac{3}{8}\left(1+\cos ^{2} \theta\right) \frac{d \sigma^{U}}{d x d \bar{x}}+\frac{3}{4} \sin ^{2} \theta \frac{d \sigma^{L}}{d x d \bar{x}} \\
& -\frac{3}{2 \sqrt{2}} \sin 2 \theta \cos \chi \frac{d \sigma^{I}}{d x d \bar{x}}+\frac{3}{4} \sin ^{2} \theta \cos 2 \chi \frac{d \sigma^{T}}{d x d \bar{x}}
\end{aligned}
$$

[ $U=$ transverse (no flip), $L=$ longitudinal, $I=\operatorname{trv} *$ long, $T=\operatorname{trv} * \operatorname{trv}$ (flip)]. If the polar and azimuthal angles are integrated out, the cross section is given by $\sigma=\sigma^{U}+\sigma^{L}$.

It is convenient to write the helicity cross sections as

$$
\frac{\beta^{3}}{\sigma_{B}} \frac{d \sigma^{j}}{d x d \bar{x}}=\frac{\alpha_{s}}{4 \pi} \frac{S^{j}+\mu^{2} N^{j}}{(1-x)(1-\bar{x})}
$$

The densities $S^{j}$ and $N^{j}$ are summarized in Table 1; $p$ is the momentum of the $\tilde{t}_{1}$ squark, $\bar{p}$ and $k$ are the longitudinal momenta of $\tilde{\tilde{t}}_{1}$ and $g$ in the $\tilde{t}_{1}$ direction, and $p_{T}$ is the modulus of the transverse $\tilde{\tilde{t}}_{1}, g$ momentum with respect to this axis [all momenta in units of the beam energy]. Since $I, T$ correspond to $\gamma, Z$ helicity flips by 1 and 2 units, they are of order $p_{T}$ and $p_{T}^{2}$, respectively. Note that the threshold suppression is absent in the $U, I, T$ components and attenuated in the leading longitudinal $L$ term as expected from eq.(3).

Fragmentation. In the limit where the gluons are emitted from fast moving squarks with small angles, the gluon radiation

$$
\tilde{t}_{1} \rightarrow \tilde{t}_{1} g
$$

can be interpreted as a perturbative fragmentation process. From the form of the differential cross section $d \sigma / d z d p_{T}^{2}$ we find in this limit for the splitting functions, in analogy to the 


\begin{tabular}{|c|c|c|}
\hline & $S^{j}$ & $N^{j}$ \\
\hline$U$ & $\frac{32}{3}(1-x)(1-\bar{x})$ & $-\frac{4}{3} p_{T}^{2} \frac{1-x}{1-\bar{x}}$ \\
$L$ & $\frac{16 \beta^{2}}{3}(1-z)$ & $\frac{4}{3}\left[p_{T}^{2} \frac{1-x}{1-\bar{x}}-\beta^{2}\left(\frac{1-x}{1-\bar{x}}+\frac{1-\bar{x}}{1-x}+2\right)\right]$ \\
$I$ & $-\frac{4 \sqrt{2}}{3} p_{T} p$ & $\frac{2 \sqrt{2}}{3} p_{T}\left(p-\bar{p} \frac{1-x}{1-\bar{x}}\right)$ \\
$T$ & 0 & $\frac{2}{3} p_{T}^{2} \frac{1-x}{1-\bar{x}}$ \\
\hline
\end{tabular}

Table 1: Coefficients of the helicity cross sections in eq.(5). The energy and momentum variables are defined in the text.

Weizsäcker-Williams [15] and Altarelli-Parisi splitting functions [16],

$$
\begin{aligned}
P\left[\tilde{t}_{1} \rightarrow \tilde{t}_{1} ; x\right] & =\frac{\alpha_{s}}{2 \pi} \frac{8}{3} \frac{x}{1-x} \log \frac{Q^{2}}{m_{\tilde{t}_{1}}^{2}} \\
P\left[\tilde{t}_{1} \rightarrow g ; z\right] & =\frac{\alpha_{s}}{2 \pi} \frac{8}{3} \frac{1-z}{z} \log \frac{Q^{2}}{m_{\tilde{t}_{1}}^{2}}
\end{aligned}
$$

As usual, $x$ and $z$ are the fractions of energy transferred from the $\tilde{t}_{1}$ beam to the squark $\tilde{t}_{1}$ and the gluon $g$ after fragmentation, respectively; $Q$ is the evolution scale of the elementary process, normalized by the squark mass rather than the QCD $\Lambda$ parameter [in contrast to the light quark/gluon sector]. As a consequence of angular-momentum conservation, the gluon cannot pick up the total momentum of the squark beam. [Similar zeros have been found for helicity-flip fragmentation functions in QED/ QCD [16, 17].]

By using the crossing rules $\{z \rightarrow 1,1 \rightarrow x\}$ and $\{1-x \leftrightarrow 1-x\}$, familiar from the analogous splitting functions in QED [18], we derive for the elementary gluon splitting process into a squark-antisquark pair

$$
g \rightarrow \tilde{t}_{1} \tilde{t}_{1}
$$

the distribution

$$
P\left[g \rightarrow \tilde{t}_{1} ; x\right]=\frac{\alpha_{s}}{2 \pi} \frac{1}{2} x(1-x) \log \frac{Q^{2}}{m_{\tilde{t}_{1}}^{2}}
$$

after adjusting color and spin coefficients properly. This splitting function is symmetric under the $\tilde{t}_{1} \leftrightarrow \tilde{t}_{1}$ exchange, i.e. $\{x \leftrightarrow 1-x\}$. The probability is maximal for the splitting into equal fractions $x=1 / 2$ of the momenta, in contrast to spinor QED/QCD where the splitting into a quark-antiquark pair is proportional to $x^{2}+(1-x)^{2}$ and hence asymmetric configurations are preferred.

The above splitting functions provide the kernels for the shower expansions in perturbative QCD Monte Carlos for $e^{+} e^{-}$annihilation such as Pythia [19] and Herwig [20]. They serve 
the same purpose in the hadron-hadron versions of these generators as well as Isajet [21]. Of course, the interpretation of the radiation processes as universal fragmentation processes becomes increasingly adequate with rising energy of the fragmenting squarks/gluons.

If the $\tilde{t}_{1}$ squark is lighter than the top quark, the lifetime will be long, $\tau \geq 10^{-20} \mathrm{sec}$, since the dominant decay channel $\tilde{t}_{1} \rightarrow t+\tilde{\chi}_{1}^{0}$ is shut off $\left[\tilde{\chi}_{1}^{0}=L S P\right]$. The decay widths corresponding to the 2-body decay $\tilde{t}_{1} \rightarrow c+\tilde{\chi}_{1}^{0}$ and 3-body slepton decays involve the electroweak coupling twice and hence will be very small [2]. As a result, the lifetime is much longer than the typical non-perturbative fragmentation time of order $1 \mathrm{fm}$ [i.e. $\left.\mathcal{O}\left(10^{-23} \mathrm{sec}\right)\right]$ so that the squark has got enough time to form $\left(\tilde{t}_{1} \bar{q}\right)$ and $\left(\tilde{t}_{1} q q\right)$ fermionic and bosonic hadrons. However, the energy transfer due to the non-perturbative fragmentation, evolving after the early perturbative fragmentation, is very small as a result of Galilei's law of inertia. Describing this last step in the hadronization process of a $\tilde{t}_{1}$ jet by the non-perturbative fragmentation function à la Peterson et al. [22] (which accounts very well for the heavyquark analogue), we find

$$
D(x)^{N P} \approx \frac{4 \sqrt{\epsilon}}{\pi} \frac{1}{x[1-1 / x-\epsilon /(1-x)]^{2}}
$$

with the parameter $\epsilon \sim 0.5 \mathrm{GeV}^{2} / m_{\tilde{t}_{1}}^{2}$. Here, $x=E\left[\left(\tilde{t}_{1} \bar{q}\right)\right] / E\left[\tilde{t}_{1}\right]$ is the energy fraction transferred from the $\tilde{t}_{1}$ parton to the $\left(\tilde{t}_{1} \bar{q}\right)$ hadron etc. The resulting average non-perturbative energy loss

$$
<1-x>^{N P} \sim \frac{2 \sqrt{\epsilon}}{\pi}\left[\log \left(\frac{1}{\epsilon}\right)-3\right]
$$

is numerically at the level of a few percent.

Monte Carlo programs for the hadronization of $\tilde{t}_{1}$ squarks link the early perturbative fragmentation with the subsequent non-perturbative hadronization. The relative weight of perturbative and non-perturbative fragmentation can be characterized by the average energy loss in the two consecutive steps. The overall retained average energy of the $\tilde{t}_{1}$ squarks factorizes into the two components,

$$
<x>=<x>^{N P}<x>^{P T}
$$

Summing up the energy loss due to multiple gluon radiation at high energies, we find in analogy to heavy-quark fragmentation 23

$$
<x>^{P T}=\left[\frac{\alpha_{s}\left(m_{\tilde{t}_{1}}^{2}\right)}{\alpha_{s}\left(E^{2}\right)}\right]^{-8 / 3 b}
$$

with $b=\left(11-2 n_{f} / 3\right)+\left(-2-n_{f} / 3\right)$ being the LO QCD $\beta$ function including the colored supersymmetric particle spectrum. At high energies, the perturbative multi-gluon radiation has a bigger impact than the final non-perturbative hadronization mechanism, e.g. $\langle x\rangle^{P T} \approx 0.93$ for a $\tilde{t}_{1}$ beam energy $E=1 \mathrm{TeV}$ and $m_{\tilde{t}_{1}}=200 \mathrm{GeV}$ as compared to $<x>^{N P} \approx 0.98$. At low energies the two fragmentation effects are of comparable size.

After finalizing the manuscript, we received a copy of Ref. 10 in which the total cross sections for squark pair production in $e^{+} e^{-}$annihilation have been discussed including squarkgluon and quark-gluino loops, yet not the gluon-jet distributions analysed in the present note. 
We thank our colleagues at the LEP2 Workshop who demanded the analysis presented here to refine the experimental stop search techniques.

\section{References}

[1] J. Ellis and S. Rudaz, Phys. Lett. B128 (1983) 248.

[2] K. Hikasa and M. Kobayashi, Phys. Rev. D36 (1987) 724.

[3] K.A. Olive and S. Rudaz, Phys. Lett. B340 (1990) 74.

[4] J. Shirai et al. (Venus), Phys. Rev. Lett. 72 (1994) 3313.

[5] R. Akers et al. (Opal), Phys. Lett. B337 (1994) 207.

[6] J.M.L. Swartz, SLAC-PUB-6710 (Nov. 1994).

[7] J. Schwinger, Particles, Sources and Fields vol. II (Addison-Wesley, New York 1973).

[8] M. Drees and K. Hikasa, Phys. Lett. B252 (1990) 127.

[9] W. Beenakker, R. Höpker, M. Spira and P.M. Zerwas, Report DESY 94-212.

[10] A. Arhrib, M. Capdequi-Peyranere and A. Djouadi, Montreal Report UdeM-GPP-94-13.

[11] A. Sommerfeld, Atombau und Spektrallinien vol. 2 (Vieweg, Braunschweig 1939).

[12] I.I. Bigi, V.S. Fadin and V. Khoze, Nucl. Phys. B377 (1992) 461.

[13] E. Laermann, K.H. Streng and P.M. Zerwas, Z. Phys. C3 (1980) 289.

[14] E. Laermann and P.M. Zerwas, Phys. Lett. B89 (1980) 225.

[15] C.F.v. Weizsäcker, Z. Phys. 88 (1934) 612; E.J. Williams, Phys. Rev. 45 (1934) 729.

[16] G. Altarelli and G. Parisi, Nucl. Phys. B126 (1977) 298.

[17] B. Falk and L.M. Sehgal, Phys. Lett. B325 (1994) 509.

[18] M.-S. Chen and P.M. Zerwas, Phys. Rev. D12 (1975) 187.

[19] T. Sjöstrand, Comp. Phys. Commun. 82 (1994) 74.

[20] G. Marchesini et al., Comp. Phys. Commun. 67 (1992) 465; B.R. Webber, CavendishHEP-94/17.

[21] H. Baer, F. E. Paige, S. D. Protopopescu and X. Tata, Report on ISAJET 7.0/ ISASUSY 1.0, FSU-HEP 930329 and UH-511-764-93.

[22] C. Peterson, D. Schlatter, I. Schmitt and P.M. Zerwas, Phys. Rev. D27 (1983) 105.

[23] I.I. Bigi, Yu.L. Dokshitser, V. Khoze, J.H. Kühn and P.M. Zerwas, Phys. Lett. B181 (1986) 157. 


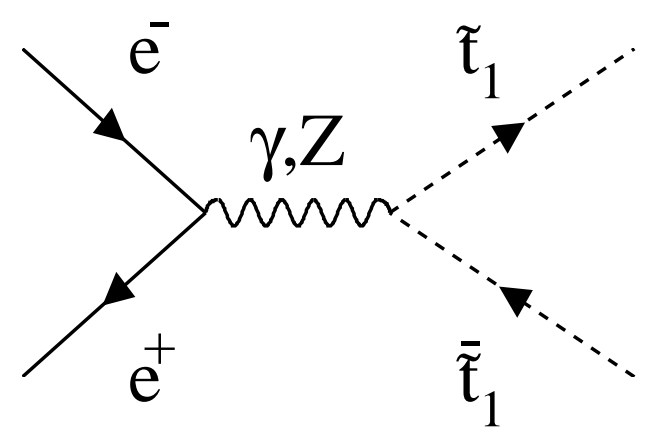

(a)
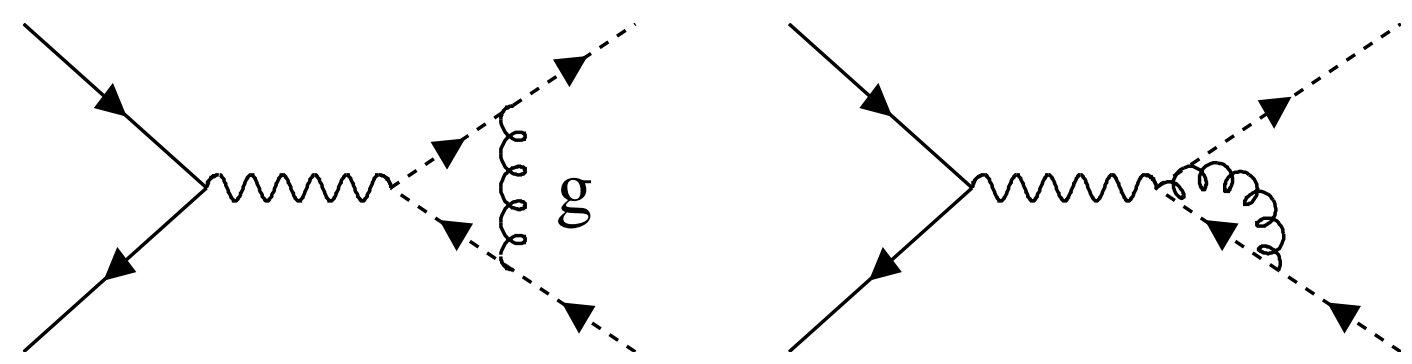

(b)
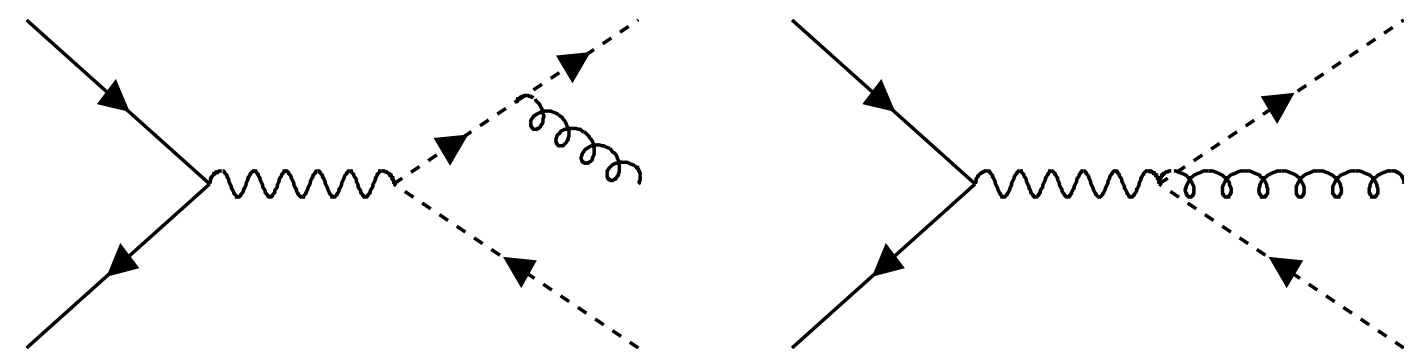

(c)

Figure 1: Generic diagrams for $\tilde{t}_{1} \overline{\tilde{t}}_{1}$ production in $e^{+} e^{-}$collisions. (a) Born level; (b) virtual QCD corrections; (c) gluon radiation. 


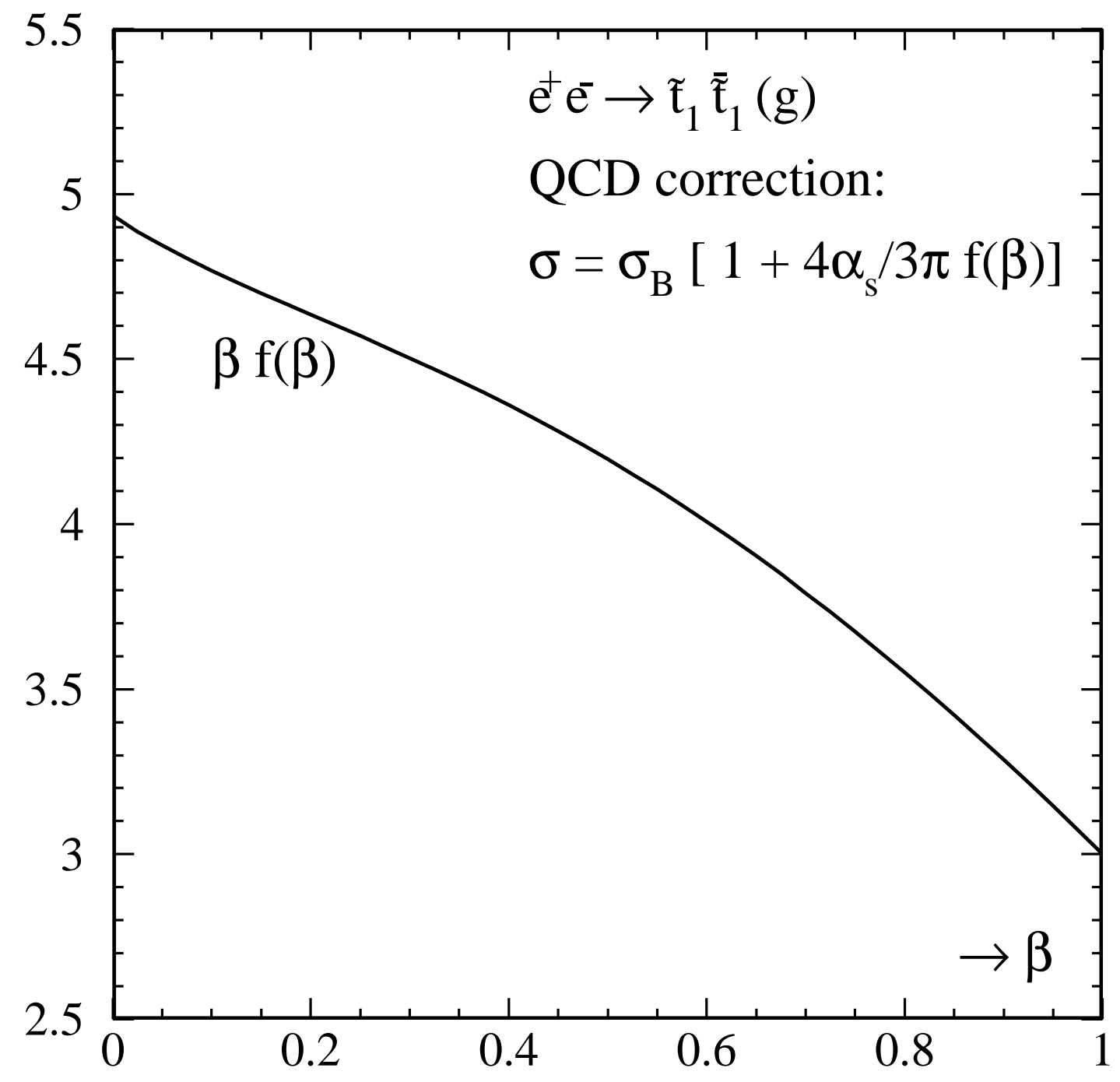

Figure 2: Coefficient of the QCD correction to the total cross section; shown is $\beta f(\beta)$, cf. eq.(3), with $\beta=\left(1-4 m_{\tilde{t}_{1}}^{2} / s\right)^{1 / 2}$. 